\title{
Optimal Sizing of Distributed Energy Resources for Integrated Microgrids using Evolutionary Strategy
}

\author{
T. Logenthiran \\ Department of ECE \\ National University of \\ Singapore \\ Singapore \\ logenthiran@nus.edu.sg
}

\author{
D. Srinivasan \\ Department of ECE \\ National University of \\ Singapore \\ Singapore \\ dipti@nus.edu.sg
}

\author{
A. M. Khambadkone \\ Department of ECE \\ National University of \\ Singapore \\ Singapore \\ eleamk@nus.edu.sg
}

\author{
T. Sundar Raj \\ Department of CHBE \\ National University of \\ Singapore \\ Singapore \\ chesrt@nus.edu.sg
}

\begin{abstract}
Optimal selection and sizing of Distributed Energy Resources (DER) is an important research problem for the advancement of distributed power systems. This paper presents detail studies on optimal sizing of DER for integrated microgrids using Evolutionary Strategy (ES). Integrated microgrid is an innovative architecture in distributed power systems, in which several microgrids are interconnected with each other for superior control and management of the distributed power systems. Right coordination among DER in microgrids, and proper harmony among the microgrids and the main distribution grid are critical challenges. Types of DER and capacities of them are needed to optimize such that proposed integrated microgrid provides reliable supply of energy at cheap cost. In this research, the problem is formulated as a nonlinear mixed-integer minimization problem which minimizes capital and annual operational cost of DER subject to a variety of system and unit constraints. Evolutionary strategy was developed for solving the minimization problem. The proposed methodology was used to design integrated microgrids for A*Star IEDS (Intelligent Energy Distribution System) project. The design results have shown that the proposed methodology provides excellent convergence and feasible optimum solution.
\end{abstract}

Keywords-Optimal sizing, Distributed energy resources, Microgrid, Integrated microgrid, Evolutionary strategy

\section{INTRODUCTION}

Microgrids are low voltage distribution networks comprising various distributed generators, storage devices, and controllable loads [1,2]. Enhanced power quality, high reliability, cheap energy cost, and reduced emissions are the important capabilities of microgrids to shift distributed power systems towards smart grid [1]. Smart grid [3,4] is a vision of future power systems, which encourages optimal design and optimal operation of power systems. Integrated microgrid [5] is a recent concept which integrates many microgrids together to achieve much benefits from them. In an integrated microgrid, each microgrid could contain different types of loads and energy sources depending on the infrastructure and nature of the area. As a result, interaction among the microgrids, and interaction between the microgrids and the main distribution grid are become crucial tasks for implementing control and management schemes. Therefore, detailed research on integrated microgrid which is a part of larger electrical

This work was supported by Science and Engineering Research Council of the Agency for Science, Technology and Research (A*STAR), Singapore IEDS programme grant R-263-000-507-305. distribution network is necessary for the design, development and control of distributed power systems.

Proper selection and sizing of distributed energy resources are some of the challenging tasks in long-term planning of distributed power systems. Diverse criteria [6,7] were considered in selecting distributed energy resources for an integrated microgrid. Some of the important criteria are load type (i.e. residential, commercial or industrial), load priority (i.e. base load, backup load or peck load shaving), operational mode (i.e. grid-connected or islanded), average load, and available distributed energy resource technologies. Once, an appropriate distributed energy resource is chosen, the subsequent questions will arise. Some of them are power rating, reliability, capital cost, installation cost, operation and management cost, method of payment, and life time of distributed energy resource. Cost-benefit of distributed energy resources can be determined by comparing the electricity price from distributed energy resources with the electricity price of the main distribution grid. In reality, cheap energy cost is not only the sole objective. The other objectives such as environmental friendliness and energy security [7] are also need to consider while planning distributed power systems.

In the literature, several research works were addressed different approaches for sizing of distributed energy resources such as wind turbines, photovoltaic systems, and batteries without applying any optimizing methodologies [8-13]. In few research works have applied optimization methodologies. For examples, tangent method $[6,13]$ is a well known approach used to size wind turbines, photovoltaic systems, and batteries for standalone hybrid wind and photovoltaic systems. Genetic algorithm [14] was employed to optimize sizes of wind turbines, photovoltaic systems, and batteries for a distribution system using genetic algorithm. The authors have proposed Evolutionary Strategy (ES) [15] for sizing of distributed energy resources for islanded microgrids. This work proved that evolutionary strategy is a promising algorithm for the problem based on the convergence characteristics and the quality of the solutions.

In this paper, the evolutionary strategy is proposed for the optimal selection and sizing of distributed energy resources for integrated microgrids. Even though decisions about the 
installation of sources and microgrids are independent from each other, and depend on the preference of the owners, distributed network operators have right to control the installation of the sources and the microgrids such that the distributed power systems meet the environmental and economic conditions according to the government and power systems.

In this paper, the problem is formulated as a nonlinear mixed-integer minimization problem which aims to reduce the sum of capital and annual operational cost of distributed energy resources subject to constraints such as desired system reliability and restrictions of generators and storage systems. A real-coded evolutionary strategy was developed to solve the minimization problem.

The remaining paper is organized as follows. Section II formulates the problem mathematically. Section III describes the models of distributed energy resources. Section IV provides the calculation procedure of reliability measures. Section V provides operational strategies of integrated microgrids based on network architecture. Section VI provides an overview of the proposed methodology. Section VII provides case studies. Section VIII provides the results and discussions. Section IX concludes the paper.

\section{PROBLEM FoRMULATION}

Fig.1 shows a schematic diagram of an integrated microgrid.

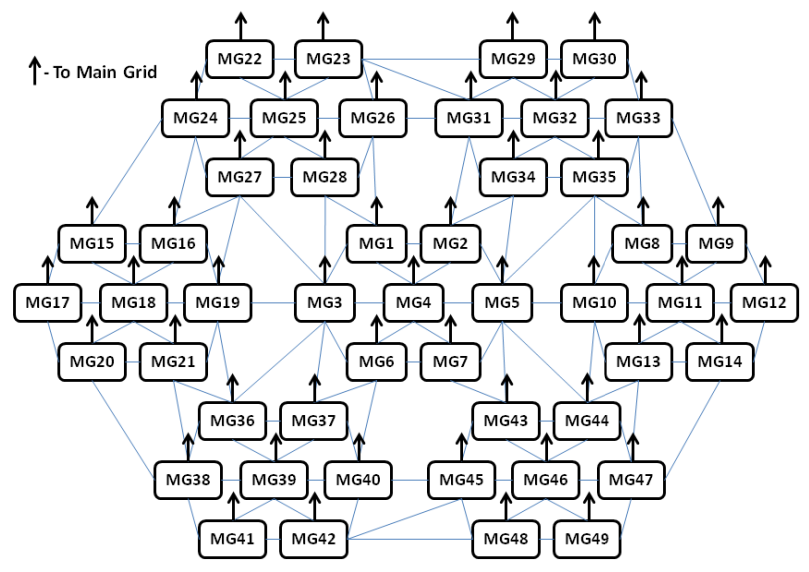

Fig.1. Schematic representation of an integrated microgrid.

The problem is mathematically formulated as follows.

Minimize,

$C_{T}=\sum_{i=1}^{N_{i}} \sum_{j=1}^{N_{j}} C_{i j} N_{i j}+M_{i j}$

where, $C_{T}$ is the total cost, $C_{i j}$ and $N_{i j}$ are the capital cost and power rating of distributed energy resource $j$ in microgrid $i$ respectively, and $M_{i j}$ is the operational and maintenance cost of distributed energy resource $j$ in microgrid $i$.
This minimization problem is subject to following constraints.

$E_{i j}^{\min } \leq E_{i j} \leq E_{i j}^{\max } \quad \forall i, j$

where, $E_{i j}^{\min }$ and $E_{i j}^{\max }$ are the minimum and maximum energy limits of distributed energy resource $j$ in microgrid $i$ respectively.

$E m_{i j}^{\min } \leq E m_{i j} \leq E m_{i j}^{\max } \quad \forall i, j$

where, $E m_{i j}^{\min }$ and $E m_{i j}^{M a x}$ are the minimum and maximum emission limits of distributed energy resource $j$ in microgrid $i$ respectively. Typically, the minimum emission limit of distributed energy resource is zero, but it is set to $E m_{i j}^{M i n}$ for the generalization of the problem formulation.

$L O L P_{i} \leq L O L P_{i}^{M} \quad \forall i$

where, $L O L P_{i}$ and $L O L P_{i}^{M}$ are the loss of load probability of microgrid $i$ and its maximum allowable limit respectively.

$L O L P \leq L O L P^{M a x}$

where, LOLP and LOLPMax are the loss of load probability of overall integrated microgrid and its maximum allowable limit respectively.

\section{Modeling OF Distributed ENERGy RESOURCES}

Distributed energy resources can be classified as controllable sources and non-controllable sources. Renewable energy sources such as photovoltaic panels and wind turbines are the major non-controllable sources. Distributed generators that use fuel resources are controllable resources. Several models are available in literature to describe the behaviours of non-controllable resources with varying complexity [18-21]. In order to choose an appropriate model, several factors such as model type, accuracy and computational effort are needed to consider $[19,20]$.

In this study, it is preferred to use less complexity model without sacrificing the accuracy because computational effort for function evaluation is the major time consuming task in this optimization. It is considered that enough fuel is available for the controllable sources which can provide any power within their limits at anytime. On the other hand, power from the noncontrollable sources depends on the meteorological data. Therefore, it is necessary to model non-controllable sources to know the available power at anytime. The models chosen for photovoltaic panel, wind turbine and composite energy storage system can be seen in [15].

The proposed sizing problem provides the best energy and power rating of composite energy storage system for each microgrid. Selection of individual energy storage elements should be done by analyzing their individual characteristics and manufacturing details. These can be also included together with this optimization if all data is available. 


\section{RELIABILITY MEASURES}

Loss of Load Probability (LOLP) is used as the reliability measure in this study. Loss of load probability is defined as a fraction of total load demand that is not supplied during simulation horizon. Calculation of loss of load probability of microgrid and integrated microgrid is given as follows.

Total energy generated at time $t$ from renewable energy sources: wind turbine and photovoltaic system can be expressed as follows.

$E_{R, t}=N_{P V} E_{P V, t}+N_{W T G} E_{W T G, t}$

where, $E_{P V, t}$ and $E_{W T G, t}$ are the energy generated from photovoltaic module and wind turbine respectively. $N_{P V}$ and $N_{W T G}$ are number of photovoltaic modules in the photovoltaic system and number of wind turbines in the wind plant respectively.

If the sum of energy generated from wind plant and photovoltaic system is less than the load demand, the short of energy $E_{N R, t}$ to satisfy the load demand is generated from other distributed generators.

$E_{G, t}=E_{R, t}+E_{N R, t}$

where, $E_{G, t}$ is the total energy generated by all sources except the composite energy storage system.

If the sum of energy generated by all distributed generators and renewable energy sources exceeds the load demand, the excess energy is used to charge composite energy storage system.

$E_{B, t}=E_{B,(t-1)}(1-\sigma)+\left[E_{G, t}-\frac{E_{L, t}}{\eta_{\text {inv }}}\right] \eta_{b a t}$

where, $E_{B,(t-1)}$ and $E_{B, t}$ is the energy stored in composite energy storage system at time $(t-1)$ and $t$ respectively, $E_{L, t}$ is the load demand, $\eta_{b a t}$ is the round-trip efficiency of composite energy storage system, $\eta_{i n v}$ is the inverter efficiency, and $\sigma$ is the self-discharge rate of composite energy storage system.

When the load demand is greater than the available energy generated, the composite energy storage system will be discharged by the amount that is needed to cover the energy deficit. This is expressed as follows.

$E_{B, t}=E_{B,(t-1)}(1-\sigma)+\left[\frac{E_{L, t}}{\eta_{i n v}}-E_{G, t}\right]$

Loss of Power Supply (LPS) is the energy deficit which is not satisfied from the energy generated from all distributed energy resources. This can be written as follows.

$L P S_{t}=E_{L, t}-\left[E_{G, t}+E_{B,(t-1)}-E_{B \min }\right] \eta_{i n v}$

where, $E_{B \min }$ is the minimum energy needed to have in composite energy storage system for its safety.

$L O L P$ is defined as the fraction of total load demand that is not supplied during simulation horizon. In other words, $L O L P$ is the ratio of summation of all $L P S_{t}$ to summation of the load demand during the simulation horizon.

$L O L P=\frac{\sum_{t=1}^{T} L P S_{t}}{\sum_{t=1}^{T} E_{L, t}}$

Interaction among microgrids is very important to identify the best power transferring among microgrids in integrated microgrids. In this research, it is modeled similar to the transportation problem [22]. Each microgrid acts as a supply center and a demand center. Therefore, dimension of power transfer matrix for an integrated microgrid with $n$ microgrids is $(n+1) \times n$, where the additional $(n+1)^{\text {th }}$ row is allocated for the main power grid. The Power Transferring Matrix $(P T M)$ is written as follows.

\section{$P T M=$}

$\left\{\begin{array}{cccccc}\text { Supply/Demand } & M G_{1} & M G_{2} & M G_{3} & \ldots & M G_{n} \\ M G_{1} & X_{11} & X_{12} & X_{13} & \ldots & X_{1 n} \\ M G_{2} & X_{21} & X_{22} & X_{23} & \ldots & X_{21} \\ M G_{3} & X_{31} & X_{32} & X_{33} & \ldots & X_{31} \\ \ldots & \ldots & \ldots & \ldots & \ldots & \ldots \\ M G_{n} & X_{n 1} & X_{n 2} & X_{n 3} & \ldots & X_{n n} \\ M G_{n+1} & X_{n+11} & X_{n+12} & X_{n+13} & \ldots & X_{n+1 n}\end{array}\right\}$

where, $X_{y z}$ is the power from microgrid $y$ to microgrid $z$.

It is considered that all microgrids have high priority to satisfy their internal loads as much as possible. Therefore, the maximum allowable power transfer limit between microgrids are given as follows.

$$
\begin{array}{cl}
0 \leq X_{y z} \leq \infty & , y=z \\
0 \leq X_{y z} \leq X_{y z}^{\max } & , y \neq z
\end{array}
$$

where, $X_{y z}^{\max }$ is the power transferring capacity of interconnection lines.

The power transferring among microgrids is optimized by Vogel's approximation (UV) method [22]. By knowing the power transferring among microgrids, available energy $E_{m, t}^{i}$ for the internal load of each microgrid can be found. For example, available energy for microgrid $z$ at hour $t$ can be found as follows.

$$
E_{m, t}^{j}=\sum_{i=1}^{N} X_{i j}
$$

where, $N$ is the number of microgrids in the integrated microgrid.

When the available energy for a microgrid is more than the internal load demand of the microgrid, composite energy storage system in the microgrid is charged as per equation (8). When the load demand is greater than the available energy, composite energy storage system is discharged to cover-up the deficit as per equation (9). The reliability measure LOLP for the integrated microgrid is proposed as the fraction of total load 
demand not supplied by the integrated microgrid during the horizon. LOLP of the integrated microgrid is written as follows.

$L O L P=\frac{\sum_{j=1}^{N} \sum_{t=1}^{T} L P S_{t}^{j}}{\sum_{j=1}^{N} \sum_{t=1}^{T} E_{L, t}^{j}} ;$

where, $L P S_{t}^{j}$ and $E_{L, t}^{j}$ are the loss of power supply at time $t$ in microgrid $j$, and the load demand at time $t$ in microgrid $j$.

\section{OPERATIONAL StRATEGIES OF INTEGRATED MicrogridS}

In this research, different operational strategies of integrated microgrids were investigated. The operational strategies depend on several factors such as architecture and interconnection among the microgrids, renewable energy potential of the regions, energy policies and financial contracts. Three different operational strategies based on the interconnection among the microgrids were studied in details, which are briefly presented as follows.

\section{A. Strategy 1: Islanded Microgrids}

In this strategy, all microgrids in the integrated microgrid are operated in islanded mode, and remain self sustainable. Schematic diagram of this strategy is shown in Fig.2.

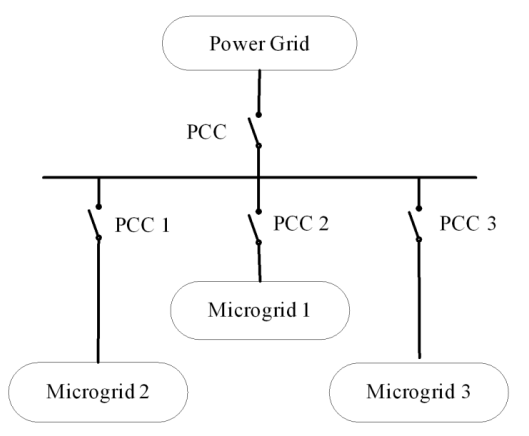

Fig. 2. Symmetric diagram of an integrated microgrid for strategy 1

Each microgrid operates independently. Therefore, power generation and energy cost purely depends on its available energy potential. For example, a microgrid with high renewable energy is expected to satisfy its local loads by renewable energy sources, and another microgrid with low renewable energy is expected to satisfy its local loads by other distributed generators as well. This results incomplete utilization of renewable energy sources. In this strategy, all microgrids act as a supply centers for their internal loads only. Therefore, the power transfer matrix for this strategy is tailored as follows.

$P T M=\left\{\begin{array}{cccc}\text { Supply/Demand } & M G_{1} & M G_{2} & M G_{3} \\ M G_{1} & X_{11} & 0 & 0 \\ M G_{2} & 0 & X_{22} & 0 \\ M G_{3} & 0 & 0 & X_{33}\end{array}\right\}$

\section{B. Strategy 2: Islanded Integrated Microgrid}

In this strategy, all microgrids are interconnected with each other. Interconnection of all microgrids provides resource sharing among them. For example, excess renewable energy available at a microgrid can be used to serve the other microgrids. Fig. 3 illustrates this operational strategy.

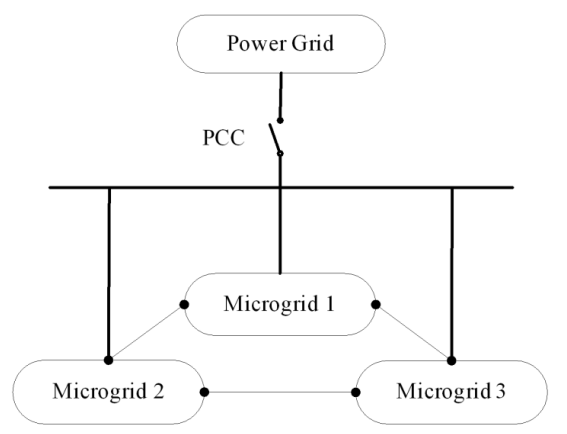

Fig.3. Symmetric diagram of an integrated microgrid for strategy 2

Power transferring among microgrids are limited by the maximum power transferring capacities of the interconnection lines among the microgrids. In this strategy, all microgrids are considered as one integrated system. The developed algorithm optimizes the total cost of the overall system. The power transfer matrix for this strategy is modified as follows.

$$
P T M=\left\{\begin{array}{cccc}
\text { Supply/Demand } & M G_{1} & M G_{2} & M G_{3} \\
M G_{1} & X_{11} & X_{12} & X_{13} \\
M G_{2} & X_{21} & X_{22} & X_{23} \\
M G_{3} & X_{31} & X_{32} & X_{33}
\end{array}\right\}
$$

\section{Strategy 3: Grid-Connected Integrated Microgrid}

Strategy 3 is a prominent architecture of integrated microgrids where, all microgrids are interconnected with each other as well as connected with the main power grid. Schematic representation of this strategy is shown in Fig.4.

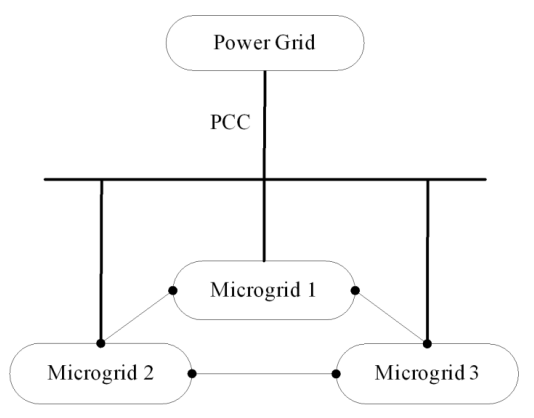

Fig. 4. Symmetric diagram of an integrated microgrid for strategy 3

The main power grid is considered as an additional entity in power transfer matrix of this strategy. The additional entity is entered with a maximum supply which is equal to the power transferring capacity of the interconnection line between the integrated microgrid and the main power grid. This strategy allows each microgrid to buy or sell power up to power transferring capacity from or to the main power grid. Similar to strategy 2, the developed algorithm for this strategy optimizes the total cost of the overall system. The power transfer matrix for this strategy is modified as follows. 


$$
\begin{aligned}
& P T M= \\
& \left\{\begin{array}{ccccc}
\text { Supply/Demand } & M G_{1} & M G_{2} & M G_{3} & M G_{E} \\
M G_{1} & X_{11} & X_{12} & X_{13} & X_{1 n} \\
M G_{2} & X_{21} & X_{22} & X_{23} & X_{21} \\
M G_{3} & X_{31} & X_{32} & X_{33} & X_{31} \\
M G_{E} & X_{E 1} & X_{E 2} & X_{E 3} & X_{E n}
\end{array}\right\}
\end{aligned}
$$

\section{Proposed Methodology}

The proposed methodology starts with identifying right design variables for integrated microgrids. The crucial design variables are number of photovoltaic modules, wind turbines, battery units, and power rating of other distributed generators for each microgrid in the integrated microgrid. The overall idea of the proposed methodology is given in Fig.5.

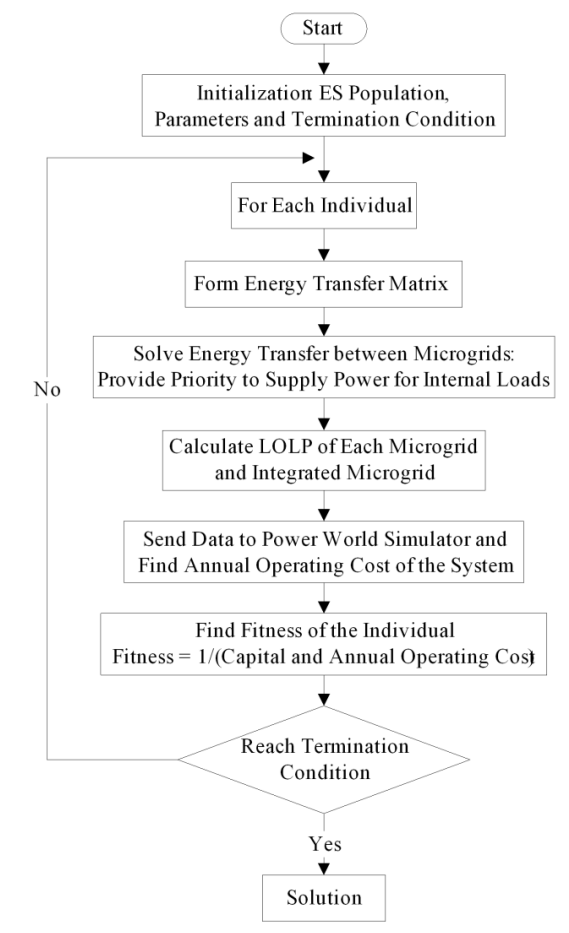

Fig. 5. Proposed methodology for optimal sizing

Evolutionary Strategy (ES) [23] is proposed and developed to solve this problem. Each chromosome is a solution associated with an objective value. The objective value is representative of the candidate solution's performance in relation to the parameter being optimized. It also reflects a candidate solution's performance with respect to other potential solutions in the space. Based on the fitness values, number of individuals is selected and genetic operators (i.e. mutation and/or recombination) are applied to generate new individuals in the next generation. The best solutions generated in one generation become the parents for the next generation.

Evolutionary strategy is an iterative method and hence the process of selection and application of genetic operators is repeated until reaching the predefined convergence criteria. After convergence, the best solution obtained is represented by the best individual so far in all generations. In this project, typical $(\mu+\lambda)$ Evolutionary Strategy (ES) is used. The basic steps of the evolutionary strategy are given as follows.

1. Generate an initial population of $\lambda$ individuals.

2. Evaluate each individual according to a fitness function.

3. Select $\mu$ best individuals called the parent population and discard the rest.

4. Apply genetic operator to create $\lambda$ off springs from $\mu$ parents.

5. Go to step 2 until a desired solution has been found or predetermined number of generations have been produced and evaluated.

Initial population of chromosomes is randomly generated within the specified practical minimum and maximum bounds of decision variables. During the simulation, each chromosome is evaluated to find out the total cost, lost of load probability, energy and emission generated from each source in each microgrid. Total cost is the objective to optimize without violating energy, emission and reliability constraints. Power World simulator [24] is a commercial software, which is used to find out the operating cost of the system. It calculates the sum of fuel cost and power transmission cost while considering power losses in the system. Power settings of each sources and details of loads at each hour are sent to Power World simulator to find out the actual operating cost of the system. A fitness function is selected such that it minimizes the total cost. The fitness function is given as follows.

$$
\text { Fitness }=\frac{1}{\text { Total Cost }}
$$

The proposed methodology was used to size distributed energy resources for integrated microgrids operating under the different operational strategies.

\section{CASE STUdIES}

The proposed methodology was used to size the distributed energy resources in the integrated microgrid of MODERN project which was carried out by National University of Singapore under IEDS program with the aid of A*STAR [25].

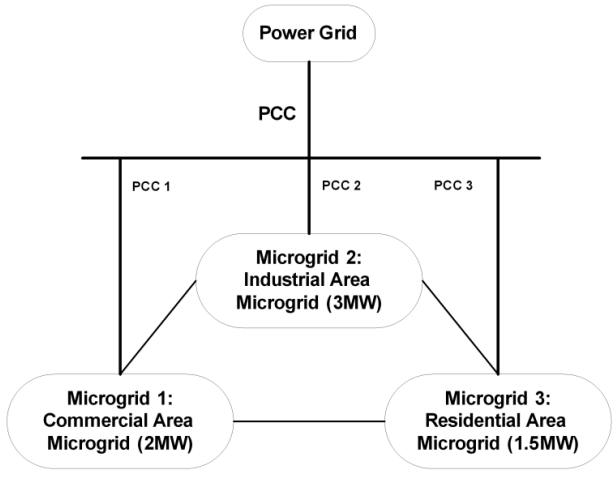

Fig. 6. Schematic diagram of the integrated microgrid

The interest is to size the resources for each microgrid optimally with reference to different operational strategies. The 
energy limits and emission limits for all distributed energy resources in the microgrids are taken from another work package of MODERN project. That work package is completely dedicated to find out possible future energy source mixes for a particular country or a region [26]. That accounts the effects of economics, renewable energy penetration, carbon emission, customer satisfaction, future uncertainties and sustainability. Schematic diagram of the integrated microgrid is shown in Fig.6. Each microgrid is built with different set of customers and sources. Microgrid $1(\mathrm{CMG})$ is for commercial types of customers, which has a peak load demand of $2 \mathrm{MW}$, whereas, Microgrid 2 (IMG) and microgrid 3 (RMG) are belonging to industrial and residential types of customers respectively, whose peak load demands are $3 \mathrm{MW}$ and $1.5 \mathrm{MW}$ respectively. Energy limits for the integrated microgrid operating under different strategies are given in Table 1.

Table 1. Average energy limits of distributed energy resources

\begin{tabular}{|c|c|c|c|c|c|c|c|c|c|}
\hline \multirow{2}{*}{ DER (\%) } & \multicolumn{3}{|c|}{ Strategy 1 } & \multicolumn{3}{c|}{ Strategy 2 } & \multicolumn{3}{c|}{ Strategy 3 } \\
\cline { 2 - 10 } & CMG & IMG & RMG & CMG & IMG & RMG & CMG & IMG & RMG \\
\hline Wind & 0.0 & 35.1 & 31.8 & 0.0 & 35.2 & 30.5 & 0.0 & 36.7 & 36.7 \\
\hline Solar & 45.3 & 35.1 & 31.8 & 49.3 & 35.2 & 30.5 & 52.1 & 36.9 & 36.9 \\
\hline Hydrogen & 24.7 & 11.7 & 2.4 & 15.8 & 11.3 & 9.8 & 3.4 & 0.0 & 0.0 \\
\hline Biomass & 10.0 & 5.1 & 0.0 & 6.4 & 4.6 & 4.0 & 10.0 & 1.3 & 1.3 \\
\hline Biodiesel & 10.0 & 6.7 & 24.3 & 10.0 & 6.7 & 25.2 & 9.5 & 0.0 & 0.0 \\
\hline Natural Gas & 3.2 & 0.0 & 0.0 & 0.0 & 0.0 & 3.3 & 0.0 & 0.0 & 0.0 \\
\hline Diesel & 6.8 & 6.3 & 9.7 & 9.5 & 6.3 & 10.0 & 0.0 & 0.0 & 0.0 \\
\hline
\end{tabular}

Emission limits are only for diesel and natural gas units. 600 tons CO2/MW-Month and 500 tons CO2/MW-Month are the emission limits of diesel and natural gas units respectively for strategy $1.90 \%$ and $75 \%$ of these values are for strategy 2 and strategy 3 respectively. These data are recommended by energy source planning by work package 1 of MODERN project [26]. Realistic of these values totally depends on the work package 1 of the project [26]. In this paper, the methodology for sizing of distributed energy resources is the main contribution. Energy limits and emission limits are just the inputs to the methodology.

Fig.7 shows the comprehensive details of the electrical network of the integrated microgrid. This network was found out by the experts in the project team. The entire network operates at a low voltage (i.e. $410 \mathrm{~V}$ ). This network may not be an optimal network structure. Identifying the optimal network architecture is another research problem, which is out of the focus of this paper.

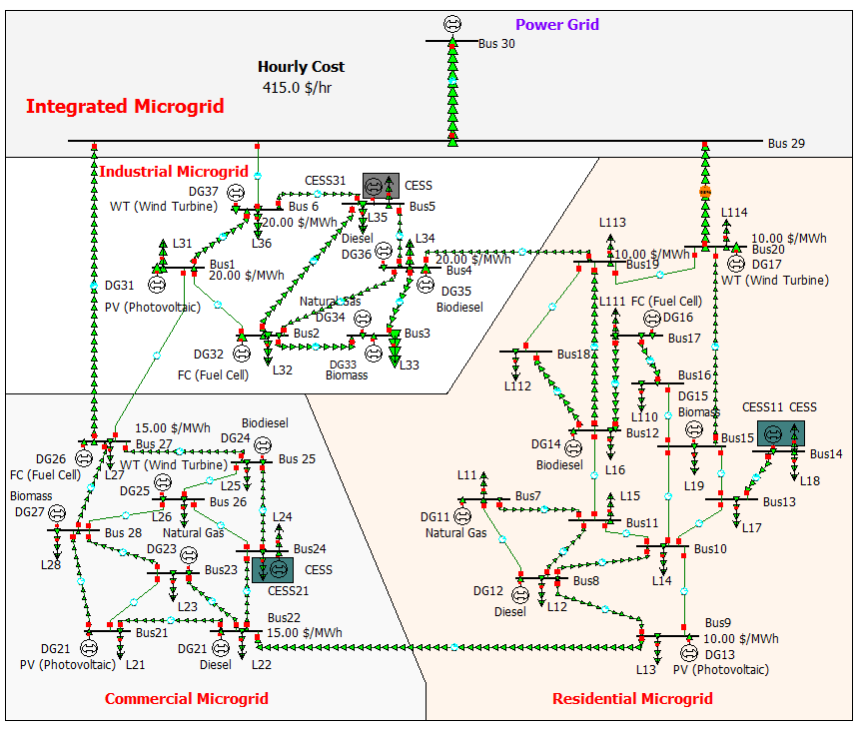

Fig.7. Electrical network diagram of the integrated microgrid
The electrical data for the interconnection links are given in Table 2. The distribution of loads and generators are provided in Table 3.

Table 2. Data of interconnection links

\begin{tabular}{|c|c|c|c|c|}
\hline $\begin{array}{c}\text { Interconnection } \\
\text { link }\end{array}$ & $\begin{array}{c}\text { Resistance } \\
(\mathrm{pu})\end{array}$ & $\begin{array}{c}\text { Reactance } \\
(\mathrm{pu})\end{array}$ & $\begin{array}{c}\text { Power Flow } \\
\text { Limit }(\mathrm{kVA})\end{array}$ & $\begin{array}{c}\text { Length } \\
(\mathrm{km})\end{array}$ \\
\hline In IMG & 0.003 & 0.01 & 500 & 5 \\
\hline In RMG & 0.00375 & 0.0125 & 500 & 2 \\
\hline In CMG & 0.0035 & 0.012 & 500 & 3 \\
\hline Among Microgrids & 0.003 & 0.01 & 500 & 7.5 \\
\hline $\begin{array}{c}\text { Between IM \& } \\
\text { Main Grid }\end{array}$ & 0.003 & 0.01 & 500 & 10 \\
\hline
\end{tabular}

In this study, $140 \mathrm{~kW}$ wind turbines and $36 \mathrm{~W}$ photovoltaic modules are considered as the basic elements of renewable energy sources. The detail characteristics and data of these units can be found in [20]. Composite energy storage system with the following specification are considered as the basic element for energy storage system. Maximum energy limit is $1000 \mathrm{kWh}$, maximum power limit is $200 \mathrm{~kW}$, and charging and discharging efficiencies are 0.9 each. The minimum state of charge is $50 \%$, and self-discharge rate per hour is $0.001 \%$. In addition, it is considered that the maximum power point trackers, battery controllers and inverters have $100 \%$ efficiencies. The number of wind turbines, photovoltaic modules and composite energy storage system required for the integrated microgrids are derived from the optimization.

Typical meteorological data for a year is taken from meteorological department [27]. Average capital cost of each distributed energy resource in the integrated microgrid can be found in [15]. Capital cost is the sum of cost associated with equipments and installation at particular location. Installation cost of distributed energy resource varies among technologies, especially less mature technologies faces wide variations. Typical installation costs are approximately $30 \%$ of the capital costs [15], however they could reach up to $100 \%$ for highly customized applications.

Operational and maintenance costs of distributed energy resources have both fixed and variable cost components. Average operational and maintenance costs for each distributed energy resources in the test system are also given in [15]. Fixed 
cost in operational and maintenance cost refers to labor cost, which is highly dependent on the operating cycle and staffing philosophy. Variable cost in operation and maintenance cost includes periodic inspection, repair works and replacement of

Table 3. Distribution of loads and generators in the integrated microgrid

\begin{tabular}{|c|c|c|c|c|c|c|c|}
\hline Bus & Microgrid & DER & Load (\%) & Bus & Microgrid & DER & Load (\%) \\
\hline 1 & IMG & PV & 35.0 & 16 & RMG & - & 5.0 \\
\hline 2 & IMG & Hydrogen & 10.0 & 17 & RMG & Hydrogen & 7.5 \\
\hline 3 & IMG & Biomass, Natural Gas & 7.5 & 18 & RMG & - & 7.5 \\
\hline 4 & $\mathrm{IMG}$ & Biodiesel, Diesel & 15.0 & 19 & RMG & - & 7.5 \\
\hline 5 & $\mathrm{IMG}$ & CESS & 20.0 & 20 & RMG & WT & 7.5 \\
\hline 6 & IMG & WT & 12.5 & 21 & CMG & PV & 15 \\
\hline 7 & RMG & Natural Gas & 7.5 & 22 & $\mathrm{CMG}$ & Diesel & 10 \\
\hline 8 & RMG & Diesel & 7.5 & 23 & $\mathrm{CMG}$ & Natural Gas & 15 \\
\hline 9 & RMG & PV & 7.5 & 24 & $\mathrm{CMG}$ & CESS & 15 \\
\hline 10 & RMG & - & 7.5 & 25 & $\mathrm{CMG}$ & Biodiesel & 10 \\
\hline 11 & RMG & - & 7.5 & 26 & CMG & WT & 10 \\
\hline 12 & RMG & Biodiesel & 7.5 & 27 & CMG & Hydrogen & 15 \\
\hline 13 & RMG & - & 10.0 & 28 & CMG & Biomass & 10 \\
\hline 14 & RMG & CESS & 5.0 & 29 & Grid & - & - \\
\hline 15 & RMG & Biomass & 5.0 & 30 & Grid & - & - \\
\hline
\end{tabular}

VIII. RESULTS AND DISCUSSIONS

Studies were carried out for yearly planning. Hourly load, wind energy and photovoltaic energy for one year were used in the studies. It is considered that these hourly data are constants in each hour. Seasonal variations of these data are not

Table 4. Distributed energy resources for the integrated microgrids

\begin{tabular}{|c|c|c|c|c|c|c|c|c|c|}
\hline \multirow[b]{2}{*}{ DER } & \multicolumn{3}{|c|}{ Strategy 1} & \multicolumn{3}{|c|}{ Strategy 2} & \multicolumn{3}{|c|}{ Strategy 3} \\
\hline & CMG & IMG & RMG & CMG & IMG & RMG & CMG & IMG & RMG \\
\hline Diesel $(\mathrm{kW})$ & 140 & 210 & 110 & 155 & 240 & 120 & - & - & - \\
\hline Natural Gas $(\mathrm{kW})$ & 220 & 330 & - & - & - & 185 & - & - & - \\
\hline Fuel Cell $(\mathrm{kW})$ & 270 & 410 & 210 & 230 & 350 & 175 & 25 & - & - \\
\hline Biodiesel $(\mathrm{kW})$ & 220 & 330 & 170 & 250 & 370 & 185 & 60 & - & - \\
\hline Biomass $(\mathrm{kW})$ & 115 & 115 & - & 110 & 160 & 80 & 75 & 110 & 55 \\
\hline Photovoltaic $(\mathrm{kW})$ & 2304 & 3420 & 1728 & 2340 & 3492 & 1764 & 2664 & 3996 & 2016 \\
\hline Wind Turbine $(\mathrm{kW})$ & - & 1400 & 700 & - & 1540 & 840 & 0 & 1540 & 840 \\
\hline Battery Bank (kW) & 800 & 1000 & 600 & 600 & 1000 & 400 & 400 & 800 & 400 \\
\hline Photovoltaic (units) & $64 \dagger$ & $95 \dagger$ & $48 \dagger$ & $65 \dagger$ & $97 \dagger$ & $49 \dagger$ & $74 \dagger$ & $111 \dagger$ & $56 \dagger$ \\
\hline Wind Turbine (units) & - & $10 \dagger$ & $5 \dagger$ & - & $11 \dagger$ & $6 \dagger$ & - & $11 \dagger$ & $6 \dagger$ \\
\hline Battery Bank (units) & $4 \dagger$ & $5 \dagger$ & $3 \dagger$ & $3 \dagger$ & $5 \dagger$ & $2 \dagger$ & $2 \dagger$ & $4 \dagger$ & $2 \dagger$ \\
\hline LOLP Achieved & 0.005 & 0.004 & 0.002 & 0.006 & 0.001 & 0 & 0 & 0.0003 & 0.0001 \\
\hline Total Cost $\left(\mathrm{x} 10^{8} \$\right)$ & 1.8532 & 2.7957 & 1.0113 & 1.4211 & 2.2344 & 1.5310 & 1.2114 & 1.9341 & 1.3120 \\
\hline
\end{tabular}

Strategy 1 has islanded microgrids without any interconnections. This strategy simplifies the problem and efforts for the optimization because it does not have any interaction among the microgrids. The proposed evolutionary strategy outperformed as expected in this strategy. The optimum total cost is $5.66 \times 10^{8}$ dollars.

Strategy 2 is an integrated microgrid which does not have any connection with the main distribution grid. This strategy aims to remain sustainable by sharing power among the microgrids. The optimum total cost is $5.18 \times 10^{8}$ dollars. This strategy has $8.5 \%$ cost benefit compared to strategy 1 . In strategy 2, the total cost at CMG and IMG is less than that needed in strategy 1 because the energy generation in $\mathrm{CMG}$ and IMG in strategy 2 is less compared to that in strategy 1 . On the other hand, the total cost of RMG increases substantially due to over exploitation of RMG to serve $\mathrm{CMG}$ and IMG energy deficits. system components, and required consumables in the plant. Average costs of various fuel sources for the year are also given in [15]. considered due to the availability of limited data. Predefined target reliability measure $L O L P_{\text {Max }}$ and $L O L P^{M a x}$ were set to 0.01 [28]. The best decisions recommended by the proposed methodology are tabulated in Table 4 for all three operational strategies.

Strategy 3 refers to an integrated microgrid with proper interconnection among the microgrids, and also connected to the main distribution grid. The optimum total cost is $4.46 \times 10^{8}$ dollars. This is $21 \%$ less than strategy 1 , and $14 \%$ less than strategy 2 respectively. This cost benefits is mainly because of power import from the main power grid, which reduces the contribution of expensive distributed energy resources in the microgrids. The results show that diesel and natural gas are not recommended because these sources are expensive when GHG penalties imposed on them as per the work package 1 [24]. This also improvises the environmental benefits by stay away from the diesel and natural gas sources. Fuel cell and biomass are also not preferred by IMG and RMG because the energy imported cost is relatively cheaper than exploiting fuel cell and biomass sources. Proposed evolutionary strategy provides good convergence for all cases. Fig. 8 shows the convergence characteristic of the evolutionary strategy for strategy 3 . 


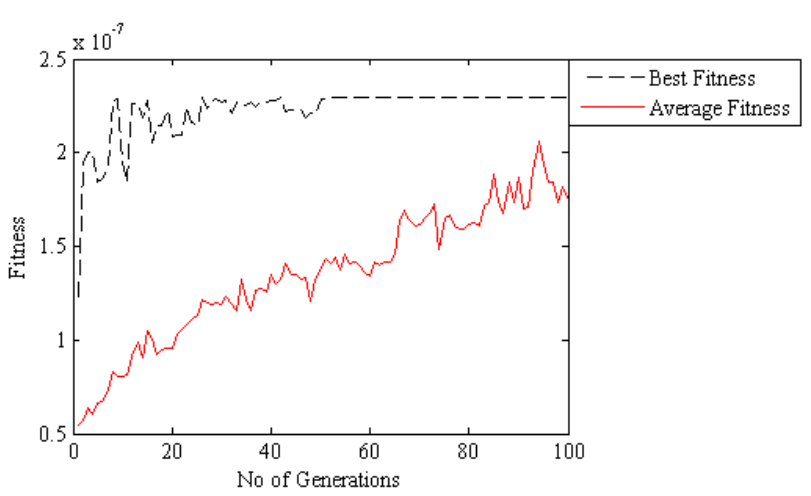

Fig.8. Convergence characteristic of ES for strategy 3

With reference to the cost measure, energy and environmental benefits, integrated microgrid adopting strategy 3 is prominent and evident to provide energy at cheap cost with enhanced energy security. Even though proposed methodology investigates three integrated microgrids with seven distributed resources at each microgrid, it can be extended to any number of distributed resources with diverse types and technologies. Flexibility is the main advantage in the proposed methodology. The chromosome structure can be modified to adopt any size of problems. Sizes of distributed generators other than the renewable energy sources and storage systems obtained from the optimization are just numbers. In order to choose available distributed generators, it is necessary to go for the nearest available ratings. It is also possible to select these from available ratings by the algorithm itself. Selection of vendors (i.e. types) for distributed generators can be added as additional genes in the chromosome if all data of each distributed generators is known.

\section{CONCLUSION}

This paper proposes a novel methodology to select and size distributed energy resources for an integrated microgrid. Even though installation of each sources and each microgrid is independent from each other, distributed network operators have right to limit the power ratings and control the installation of the sources and the microgrids such that the distributed power systems meet the environmental and economic limits according to the rules and policies of government and power systems. In this paper, the problem is formulated as a nonlinear mixed integer optimization problem which optimizes using an evolutionary strategy. The energy limits, emission limits and desired $L O L P$ were dealt as hard constraints while calculating fitness of chromosomes. Three different operational strategies of integrated microgrids were considered in the case studies. Outcomes of the studies confirmed the workability of the proposed methodology. As the proposed methodology is flexible, it is extendable for planning of any power system.

\section{REFERENCES}

[1] R.H. Lasseter, A. Akhil, C. Marnay, J. Stephens, J. Dagle, R. Guttromson, A. Meliopoulous, R. Yinger, and J. Eto, "The CERTS Microgrid Concept," White paper for Transmission Reliability Program, Office of Power Technologies, U.S. Department of Energy, Rep.LBNL$50829,2002$.
[2] MICROGRIDS: Large Scale integration of Micro-Generation to low Voltage Grids, EU contact ENK5-CT-2002-00610, Technical Annex, 2002.

[3] P. Agrawal, "Overview of DOE Microgrid Activities," Symposium on Microgrid, Montreal, 2006.

[4] Q. Li, and M. Zhou, "The future-oriented grid-smart grid," Journal of Computers , pp.98-105, 2011.

[5] T. Logenthiran, D. Srinivasan, and A.M. Khambadkone, "Multi-agent system for energy resource scheduling of integrated microgrids in a distributed system," EPSR, pp.138-148, 2011.

[6] H.L. Willis, and W.G. Scott, Distributed Power Generation: Planning and Evaluation, Marcel Dekker, New York, 2000.

[7] Available at: http://www.energy.ca.gov/distgen/

[8] B.S. Borowy, and Z.M. Salameh, "Methodology for Optimally Sizing the Combination of a Battery Bank and PV Array in a Wind/PV Hybrid System," IEEE Transaction on Energy Conversion, pp.367-375, 1996.

[9] M.A. Habib, S.A.M. Said, M.A. El-Hadidy, and A.I. Zaharna, "Optimization Procedure of Hybrid Photovoltaic Wind Energy System," Journal of Energy, pp.919-929, 1999.

[10] J.K. Kaldellis, "Parametric Investigation Concerning Dimensions of a Stand-alone Wind-Power System," Applied Energy, pp.35-50, 2004.

[11] B. Ai, H. Yang, H. She, and X. Liao, "Computer-aided Design of PV/wind Hybrid System," Renewable Energy, pp.1491-1512, 2003.

[12] M.A. Elhadidy, and S.M. Shaahid, "Optimal Sizing of Battery Storage for Hybrid (wind+diesel) Power Systems," Renewable Energy, pp.77-86, 1999.

[13] M. Al-Ashwal, and I.S. Moghram, "Proportion Assessment of Combined PV-Wind Generating Systems," Renewable Energy, pp. 43-51, 1997.

[14] D. Xu, L. Kang, and B. Cao, "Optimal Sizing of Standalone Hybrid Wind/PV Power Systems Using Genetic Algorithms," IEEE Canadian Conference on ECE, pp.1705-1708, 2005.

[15] T. Logenthiran, D. Srinivasan, A.M. Khambadkone, and T. Sundar Raj, "Optimal Sizing of an Islanded Microgrid Using Evolutionary Strategy," IEEE PMAPS, pp.12-17, 2010.

[16] H. Jiayi, J. Chuanwen, and X. Rong, "A review on distributed energy resources and MicroGrid," Renewable and Sustainable Energy Reviews, pp.2465-2476, 2008.

[17] N. Hatziargyriou, H. Asano, R. Iravani, and C. Marnay, "Microgrids," IEEE Power and Energy Magazine, pp.78-94, 2007.

[18] F. Lasnier, and T.G. Ang, Photovoltaic Engineering Handbook, IOP Publishing Ltd, Adam Hilger, New York, 1990.

[19] R. Chedid, and S. Rahman, "Unit sizing and control of hybrid wind-solar power systems," IEEE Trans. on Energy Conversion, pp.79-85, 1997.

[20] T. Logenthiran, and D. Srinivasan, "Short Term Generation Scheduling of a Microgrid," IEEE TENCON, pp.1-6, 2009.

[21] T. Ackermann, Wind Power in Power Systems, John Wiley \& Sons, Ltd, Royal Institute of Technology, Stockholm Sweden, 2005.

[22] S.T. Rachev,and L. Ruschendorf, Mass Transportation Problems, Springer-Verlag, 1998.

[23] T. Back, D. Fogel, and Z. Michalewicz, Handbook of evolutionary computation, IOP Publishing and Oxford University Press, New York, 1997.

[24] Available at: http://www.powerworld.com/.

[25] Available at: http://www.a-star.edu.sg/.

[26] T. Sundar Raj, and I.A. Karimi, "Advancements in Energy Mix planning for power grid applications under diverse energy policies," International Symposium on DOCCP, 2010.

[27] Available

at: http://courses.nus.edu.sg/course/geomr/front/fresearch/metstation

[28] J. Panida, and S. Chanan, "Comparison of Simulation Methods for Power System Reliability Indexes and their Distributions," IEEE trans. on Power Systems, pp. 486-493, 2008. 